\title{
Dementia onset, incidence and risk in type 2 diabetes: a matched cohort study with the Fremantle Diabetes Study Phase I
}

\author{
Wendy A. Davis ${ }^{1}$ - Renate R. Zilkens ${ }^{2}$ - Sergio E. Starkstein ${ }^{3} \cdot$ Timothy M. E. Davis $^{1}$ • \\ David G. Bruce ${ }^{1,4}$
}

Received: 12 April 2016/Accepted: 19 September 2016/Published online: 7 October 2016

(C) Springer-Verlag Berlin Heidelberg 2016

\begin{abstract}
Aims/hypothesis The study aimed to assess the incidence, age of onset, survival and relative hazard of dementia in wellcategorised community-based patients with type 2 diabetes compared with a matched cohort of individuals without diabetes.

Methods A longitudinal observational study was undertaken involving 1291 participants with type 2 diabetes from the Fremantle Diabetes Study and 5159 matched residents without documented diabetes. Linkage with health-related databases was used to detect incident dementia. Relative hazards were assessed using both cause-specific and subdistribution proportional hazards models.

Results During $13.8 \pm 5.8$ years of follow-up, incident dementia occurred in $13.9 \%$ and $12.4 \%$ of the groups of participants with and without diabetes, respectively $(p=0.15)$. With type 2 diabetes, the incidence of dementia was higher (incidence rate ratio [IRR] 1.28, 95\% CI 1.08, 1.51), as was the competing risk of death (IRR $1.50,95 \%$ CI 1.38, 1.64). The ages when dementia was first recorded and when death with dementia
\end{abstract}

Electronic supplementary material The online version of this article (doi:10.1007/s00125-016-4127-9) contains peer-reviewed but unedited supplementary material, which is available to authorised users.

David G. Bruce

David.Bruce@uwa.edu.au

1 School of Medicine and Pharmacology, The University of Western Australia, PO Box 480, Fremantle, WA 6959, Australia

2 Centre for Population Health Research, School of Public Health, Curtin University of Technology, Perth, WA, Australia

3 School of Psychiatry and Clinical Neurosciences, The University of Western Australia, Fremantle Hospital, Fremantle, WA, Australia

4 School of Medicine and Pharmacology, Fremantle Hospital, PO Box 480, Fremantle, Western Australia 6959, Australia occurred were both earlier with diabetes, by 1.7 (95\% CI 0.6 , $2.9)$ and 2.3 (95\% CI 1.1, 3.6) years, respectively (both $p \leq 0.004)$. Type 2 diabetes was associated with an adjusted subdistribution HR of 1.18 (95\% CI 1.00, 1.39), and a causespecific HR of $1.51(95 \%$ CI $1.27,1.78)$ for all-cause dementia. Conclusions/interpretation Type 2 diabetes is associated with an increased incidence of dementia, and dementia onset occurs at a younger age. The relative hazards of both dementia and premature mortality are increased and, as a consequence, the increased risk of dementia in type 2 diabetes is not as marked as suggested by cause-specific HRs.

Keywords Case cohort study · Dementia $\cdot$ Longitudinal study · Type 2 diabetes

\author{
Abbreviations \\ CCI Charlson Comorbidity Index \\ csHR Cause-specific HR \\ FDS1 Fremantle Diabetes Study Phase I \\ HMDS Hospital Morbidity Data System \\ IQR Interquartile range \\ IRR Incident rate ratio \\ MHIS Mental Health Information System \\ sdHR Subdistribution HR \\ WADLS Western Australian Data Linkage System
}

\section{Introduction}

There is considerable evidence from longitudinal observational studies that type 2 diabetes increases the risk of all-cause dementia, Alzheimer's disease and vascular dementia [1-4]. In two recent meta-analyses, estimates of RR associated with type 2 diabetes were 1.51 and 1.73 , respectively, for all-cause 
dementia, 1.46 and 1.56 for Alzheimer's disease, and 2.48 and 2.27 for vascular dementia [2, 3]. The importance of this risk association is underscored by the already high and increasing global prevalence of type 2 diabetes [5] and the potential for maintenance of cognitive function through effective prevention and treatment of diabetes $[6,7]$.

There are, however, several reasons why research on this topic should continue. First, the epidemiology of both conditions may be changing. In several European countries, the number of people with dementia is stabilising despite an ageing population [8-10], possibly because of improvements in education, housing and cardiovascular health [10]. In diabetes, improvements in clinical care have been accompanied by reduced rates of complications and cardiovascular disease $[11,12]$. How these two phenomena might affect the incidence of dementia in type 2 diabetes is unknown, but possible scenarios include an increased risk if more individuals with type 2 survive into old age or a reduction in risk if there are further improvements in diabetes care.

Second, there are grounds to suggest that published studies could have provided biased estimates of the risk of dementia in type 2 diabetes. It has been recommended that estimates of the population risk of age-related diseases should take into account premature mortality that may intervene before the disease of interest develops [13, 14]. Essentially, in conditions with a high mortality, the competing event of death can obscure the potential risk of dementia because individuals die before they can develop dementia. This is germane in studies of type 2 diabetes in which premature mortality is high [15]. Importantly, taking account of such competing risks tends to produce lower estimates of the magnitude of risk attributed to a specific condition, especially where the duration of follow-up is long $[13,14]$. Perhaps unbeknown to the authors of the prior papers, they have estimated the cause-specific HR (csHR) which may not reflect the actual increased risk that an individual with type 2 diabetes experiences [2-4]. This hypothesis is supported by a recent study in which the csHR for cognitive decline in diabetes was higher than the subdistribution HR (sdHR), which included not only the association of diabetes with cognitive decline, but also the association of diabetes with the competing event of death that prevented individuals experiencing cognitive decline [16].

Third, relatively minor changes in incidence rates have a large impact on future dementia projections in population models [17]. However, despite the large number of studies that have explored the association between type 2 diabetes and dementia, relatively few have examined the natural history of dementia including age of onset and survival. In a recent large population-based investigation of individuals with dementia in the state of Western Australia, we reported that patients with comorbid diabetes had an onset approximately 2 years earlier and a shorter survival than those without diabetes [18]. In other studies, vascular dementia occurred at an earlier age in diabetes [19] and survival was also shorter when Alzheimer's disease was accompanied by diabetes [20].

The Fremantle Diabetes Study Phase I (FDS1) is a longitudinal, community-based cohort study of known diabetes involving a representative patient sample from an urban Australian community. The participants were recruited between 1993 and 1996 and followed with annual face-to-face clinical assessments for a further 5 years [21]. We have previously reported risk factor data for dementia and cognitive decline in middle-aged and older subsamples of FDS1 survivors with type 2 diabetes [22-24]. Outcome data sources in FDS1 include the Western Australian Data Linkage System (WADLS), an internationally renowned, validated, population-based data linkage system that creates links between a number of Western Australia state health administrative datasets [25]. In the present study we used the WADLS to identify incident patients with dementia between recruitment up until 2012 in all FDS1 participants with type 2 diabetes and in a large (4:1) matched sample of non-diabetic residents from the same community, with the aim of comparing the age of dementia onset (using age at index dementia record as a proxy), age at death, length of survival and incidence of dementia in those with and without prior diabetes. We also compared relative hazards obtained with and without consideration of the competing risk of death.

\section{Methods}

Patients FDS1 is a longitudinal observational cohort study of residents with diabetes from a postcode-defined urban community of 120,097 people in the state of Western Australia. Descriptions of recruitment, sample characteristics, including classification of diabetes type, and details of non-recruited patients have been published elsewhere [21]. Of 2258 residents with diabetes identified between 1993 and 1996, 1426 (63\%) were recruited to the FDS1 and 1296 had type 2 diabetes. Eligible residents who declined participation were a mean 1.4 years older than the participants, but their sex distribution, the proportion with type 2 diabetes and their use of blood glucose-lowering therapies were similar. Annual assessments, comprising a comprehensive questionnaire, physical examination and fasting blood and urine sample collection, continued until 2001, but the collection of morbidity and mortality data continues through health service linkages using the WADLS [25]. The FDS1 protocol was approved by the Human Rights Committee at Fremantle Hospital and all individuals gave informed consent before participation. Data linkage was approved by the WA Department of Health Human Research Ethics Committee. 
Comparison cohort To select a comparison cohort, we used the WADLS to identify matched adults without diabetes for each recruited FDS1 adult participant. Using the electoral roll, four age-, sex- and postcode-matched residents were randomly selected from the FDS1 catchment area for each FDS1 participant at the time of FDS1 study entry. Voting is compulsory for all Australians aged 18 years and over in Federal and State elections, and thus all adults resident in the FDS1 catchment area should be listed on the electoral roll. The requirement for postcode matching was based on the fact that there are differences in socioeconomic and migrant status between residential districts within the catchment area. The individuals without diabetes had not been coded as having diabetes at any time on any Western Australia health database, before, during or after the follow-up period. Five residents without diabetes died just before entry into FDS1 and were therefore excluded. Matches could not be made for five young and four elderly participants, and they were also excluded. This left 1291 (99.6\%) FDS1 participants with type 2 diabetes who were matched with 5159 residents without diabetes.

Dementia case and comorbidity ascertainment All hospital admissions, contacts with mental health services and deaths in Western Australia are recorded in the WADLS [25], and the combination of databases has been shown to improve the identification of individuals with dementia [26-28]. The WADLS was used to determine prevalent dementia status at study entry from ICD coding from 1982 and incident dementia to the end of June 2012. The number with dementia included all patients diagnosed and registered on any of these registers and was defined using the following ICD-9-CM (www.icd9data.com/2007/Volume1) and ICD-10-AM (www.who.int/classifications/icd/en/) codes: Alzheimer's disease (331.0, F00, G30), vascular dementia (290.4, F01), unspecified dementia (290.0, 290.1, 290.2, 290.3, 294.2, 331.2, F03, G31.1) and other dementia (046.1, 291.2, 292.82, 294.1, 331.1, 331.11, 331.19, 331.82, 797, A81.0, F02, F05.1, F10.27, F10.97, G31.0, G31.10, G31.09. G31.83).

A diagnostic dementia hierarchy was used to allocate dementia type to the participants with more than one dementia code in their records [27]: Huntington's and CreutzfeldtJakob diseases took precedence over all other dementias, followed by Alzheimer's disease, vascular dementia, frontotemporal dementia and Parkinson's dementia. If the code for unspecified dementia was reported in addition to a more specific diagnosis, the latter was used. Dementia subdiagnoses were those documented by the wide range of treating physicians from both specialist and general settings. Because the diagnostic accuracy of these subdiagnoses may be low, we explored associations for all-cause dementia as the main endpoint but also investigated subdiagnoses (unspecified dementia, Alzheimer's disease and vascular dementia) for comparison purposes.

The Mental Health Information System (MHIS) started as a register of inpatients in psychiatric hospitals in 1966, expanded in the 1970s to include all other hospitals and community mental health services, and since 1980 has covered all outpatient and community-based contacts with public mental health clinics and all inpatient admissions to private or public hospitals in the state where a diagnosis of mental health disorder or self-harm has been made or where contact has occurred with a specialist psychiatric service [29]. The Hospital Morbidity Data System (HMDS) was established in 1970 and collects comprehensive data on all public and private hospital admissions in Western Australia. All deaths are registered in the Western Australia Death Registry.

The HMDS was used to determine pre-existing comorbidity during the 5 years prior to study entry, excluding dementia, diabetes and diabetic complications, using the Charlson Comorbidity Index (CCI) [30]. This includes history of myocardial infarction, congestive heart failure, peripheral vascular disease, cerebrovascular disease, chronic pulmonary disease, rheumatic disease, peptic ulcer disease, hemiplegia or paraplegia, renal disease, liver disease and cancer. We extracted and included a history of schizophrenia from the HMDS and MHIS databases given its associations with diabetes and dementia [28, 31].

Data analysis The computer packages IBM SPSS Statistics 22 (IBM, Armonk, NY, USA.) and STATA IC 13 (StataCorp LP, College Station, TX, USA) were used for statistical analyses. Data are presented as proportions, mean $\pm \mathrm{SD}$, geometric mean (SD range) or, in the case of variables that did not conform to a normal or log-normal distribution, median and interquartile range (IQR). For independent samples, two-way comparisons for proportions were analysed by Fisher's exact test, for normally distributed variables by Student's $t$ test, and for non-normally distributed variables by the Mann-Whitney $U$ test. FDS1 participants and matched residents without diabetes were followed from study entry to first record of any dementia, death or 30 June 2012, whichever came first.

The age at the index dementia record was taken as a proxy for the age of onset of dementia, also used to assess survival in those who died with dementia. Ten-year age- and sex-specific incident rates of all-cause dementia, Alzheimer's disease, vascular dementia and unspecified dementia and all-cause deaths for the FDS1 cohort were compared with those derived for the comparison group without diabetes, and incident rate ratios (IRRs) were calculated. Ten-year age groups starting at 55 years of age were selected owing to the older age of people with dementia. Cox proportional hazards models were used to generate cause-specific HRs (csHRs), while Fine and Gray competing risk models [32] generated subdistribution HRs 
(sdHRs) for all-cause dementia, Alzheimer's disease, vascular dementia and unspecified dementia in those with vs without type 2 diabetes, adjusting first for age as the time scale, and additionally for age at study entry, sex, CCI score and schizophrenia. Cox proportional HRs for all-cause death were also assessed. An increase in the csHR for dementia does not necessarily translate into an increase in the relative hazard as this will depend on the competing event of death. Given that the HR for all-cause death is higher in type 2 diabetes, one would expect the sdHR for dementia to be lower because individuals are likely to die before the development of dementia [32, 33]. Owing to the presence of covariates strongly associated with age, age was used as the time scale with left truncation of age at study entry [34]. A two-tailed significance level of $p<0.05$ was used throughout. The proportional hazards assumption was checked using Schoenfeld residuals and the $\log _{e}$-minus- $\log _{e}$ curves for Cox models, and the $\log _{e}-$ minus- $\log _{e}$ curves for the Fine and Gray models.

\section{Results}

Baseline characteristics and incident dementia At study entry, the cohort with type 2 diabetes and the cohort without diabetes were closely matched on age $(64.0 \pm 11.2$ vs 64.0 \pm 11.2 years, $p=0.91)$ and $\operatorname{sex}(48.7 \%$ male sex in both groups), and the prevalence of dementia was similar (diabetes 5/1291 [0.4\%], non-diabetes 27/5159 [0.5\%], $p=0.66)$. After exclusion of prevalent dementia, 179/1286 (13.9\%) of the cohort with type 2 diabetes and 636/5132 (12.4\%) of the cohort without diabetes were recorded with incident dementia during follow-up $(p=0.15)$. With prevalent dementia included, there were $708(54.8 \%)$ and $2122(41.1 \%)$ deaths in those with and without diabetes, respectively $(p<0.001)$, of whom $151(21.3 \%)$ and $543(25.9 \%)$ died with dementia $(p=0.014)$

The duration of type 2 diabetes at study entry was a median of 4.0 (IQR 1.0-9.0) years. Hospitalisations were significantly more frequent in the FDS1 cohort in the 5 years prior to study entry (median 1 [IQR $0-3$ ] vs 1 [IQR $0-2], p<0.001$ ), but there was no significant difference by diabetes status with respect to the source of the index dementia record $(14.0 \%$ and $19.2 \%$, respectively, in individuals with vs without diabetes from the MHIS, $76.5 \%$ vs $69.7 \%$ from the HMDS, and $9.5 \%$ vs $11.2 \%$ from death certification, $p=0.19)$. Sex and comorbidity were both associated with the index dementia record: men had a younger age than women at both the index dementia record and death (both $p<0.001$ ), and participants with a CCI score of 3-13 at study entry were significantly younger at the index dementia record than those with a CCI score of less than $3(p=0.017)$.
Age-specific incidence rates of dementia (all-cause, Alzheimer's disease, vascular dementia and unspecified dementia) in 10-year age groups from age 55 years onwards, together with the respective IRRs, are presented in Table 1. Type 2 diabetes was associated with a significantly higher incidence rate of all-cause dementia (IRR 1.28 overall) that was significant in most age groups and higher in younger age groups (IRR 4.19, 1.64, 1.43 and 1.43 in the 55-64, 65-74, 75-84 and 85+ year age groups respectively). The IRRs were significantly increased for unspecified dementia in type 2 diabetes, non-significantly increased for vascular dementia and not increased for Alzheimer's disease. The IRR for all-cause death in participants without dementia at baseline was significantly increased in type 2 diabetes (by $50 \%$ overall, significant for all age groups and higher in younger age groups; Table 1).

Dementia onset and survival To further investigate the increased incidence of dementia in type 2 diabetes, we compared ages of dementia onset, death and survival with dementia (Table 2). In type 2 diabetes, the index dementia record occurred at a significantly younger age (by $1.7[95 \%$ CI $0.6,2.9$ ] years, $p=0.004$ ) than in the non-diabetes cohort. During follow-up, $146(81.6 \%)$ people with dementia and type 2 diabetes and $525(82.6 \%)$ people with dementia and no diabetes died. Those with type 2 diabetes died a mean 2.3 $(95 \%$ CI $1.1,3.6)$ years $(p<0.001)$ earlier than those without diabetes, and median survival was a non-significant 0.6 years less $(p=0.08)$. Similar findings were seen for Alzheimer's disease, vascular dementia and unspecified dementia. Type 2 diabetes was associated with a non-significant earlier age at onset of dementia for all three subtypes, a significant earlier age at death for all subtypes and shorter survival that was significant only for Alzheimer's disease.

Diabetes-associated dementia risk The cause-specific and subdistribution HRs for all-cause dementia are presented in Table 3, and the HRs for the competing event of death in Table 4. The equivalent data for the dementia subtypes are presented in electronic supplementary material (ESM) Table 1. The proportional hazards assumption was met for type 2 diabetes status for both the cause-specific and subdistribution proportional hazards models for all-cause dementia and dementia subtypes ( $p \geq 0.07$ for Cox models). During 88,488 (13.8 \pm 5.8 years) patient-years of follow-up, type 2 diabetes was associated with an sdHR of 1.09 (95\% CI $0.92,1.29)$ and a csHR of 1.48 (95\% CI 1.26, 1.75) for all-cause dementia. With adjustment for age, sex and comorbidities, these relative hazards increased in both models, to $1.18(95 \% \mathrm{CI} 1.00,1.39)$ for the sdHR and $1.51(95 \% \mathrm{CI}$ $1.27,1.78)$ for the csHR. The cumulative incidence function curves for incident dementia among those with type 2 diabetes vs no diabetes adjusted for age, sex and comorbidities are 
Table 1 Age-specific incidence rate per 100,000 patient-years and IRR for residents with diabetes (FDS1) vs matched residents without diabetes of index all-cause dementia and subtypes and all-cause deaths

\begin{tabular}{|c|c|c|c|c|c|}
\hline \multirow[t]{2}{*}{ Variable } & \multicolumn{5}{|l|}{ Age group (years) } \\
\hline & $55-64$ & $65-74$ & $75-84$ & $85+$ & $55+$ \\
\hline \multicolumn{6}{|l|}{ All-cause dementia } \\
\hline FDS1: with dementia $(n)$ & 4 & 29 & 86 & 60 & 179 \\
\hline FDS1: years & 3430.3 & 5796.1 & 4188.1 & 830.9 & $14,245.4$ \\
\hline FDS1: IR & 116.6 & 500.3 & 2053.5 & $73,220.8$ & 1256.6 \\
\hline Non-diabetic: with dementia $(n)$ & 4 & 76 & 290 & 266 & 636 \\
\hline Non-diabetic: years & $14,380.1$ & $24,966.4$ & $20,159.1$ & 5250.4 & $64,755.9$ \\
\hline Non-diabetic: IR & 27.8 & 304.4 & 1438.6 & 5066.3 & 982.2 \\
\hline $\operatorname{IRR}(95 \% \mathrm{CI})$ & $4.19(0.78,22.51)$ & $1.64(1.03,2.55)$ & $1.43(1.11,1.82)$ & $1.43(1.06,1.89)$ & $1.28(1.08,1.51)$ \\
\hline \multicolumn{6}{|l|}{$\mathrm{AD}$} \\
\hline FDS1: with AD (n) & 1 & 11 & 31 & 14 & 57 \\
\hline FDS1: years & 3430.3 & 5796.1 & 4188.1 & 830.9 & $14,245.4$ \\
\hline FDS1: IR & 29.2 & 189.8 & 740.2 & 1684.9 & 400.1 \\
\hline Non-diabetic: with AD $(n)$ & 2 & 38 & 138 & 90 & 268 \\
\hline Non-diabetic: years & $14,380.1$ & $24,966.4$ & $20,159.1$ & 5250.4 & $64,755.9$ \\
\hline Non-diabetic: IR & 13.9 & 152.2 & 684.6 & 1714.2 & 413.9 \\
\hline $\operatorname{IRR}(95 \% \mathrm{CI})$ & $2.10(0.04,40.26)$ & $1.25(0.57,2.49)$ & $1.08(0.71,1.60)$ & $0.98(0.52,1.74)$ & $0.97(0.71,1.29)$ \\
\hline \multicolumn{6}{|l|}{$\mathrm{VaD}$} \\
\hline FDS1: with $\operatorname{VaD}(n)$ & 0 & 5 & 10 & 5 & 20 \\
\hline FDS1: years & 3430.3 & 5796.1 & 4188.1 & 830.9 & $14,245.4$ \\
\hline FDS1: IR & 0 & 86.3 & 238.8 & 601.8 & 140.4 \\
\hline Non-diabetic: with $\operatorname{VaD}(n)$ & 1 & 5 & 29 & 19 & 54 \\
\hline Non-diabetic: years & $14,380.1$ & $24,966.4$ & $20,159.1$ & 5250.4 & $64,755.9$ \\
\hline Non-diabetic: IR & 7.0 & 20.0 & 143.9 & 361.9 & 83.4 \\
\hline $\operatorname{IRR}(95 \% \mathrm{CI})$ & 0 & $4.31(0.99,18.72)$ & $1.66(0.72,3.50)$ & $1.66(0.49,4.60)$ & $1.68(0.95,2.86)$ \\
\hline \multicolumn{6}{|l|}{ Unspecified dementia } \\
\hline FDS1: with unspecified dementia $(n)$ & 3 & 10 & 38 & 34 & 85 \\
\hline FDS1: years & 3430.3 & 5796.1 & 4188.1 & 830.9 & $14,245.4$ \\
\hline FDS1: IR & 87.5 & 172.5 & 907.3 & 4092.5 & 596.7 \\
\hline Non-diabetic: with unspecified dementia $(n)$ & 0 & 27 & 103 & 116 & 246 \\
\hline Non-diabetic: years & $14,380.1$ & $24,966.4$ & $20,159.1$ & 5250.4 & $64,755.9$ \\
\hline Non-diabetic: IR & 0 & 108.1 & 510.9 & 2209.4 & 379.9 \\
\hline $\operatorname{IRR}(95 \% \mathrm{CI})$ & - & $1.60(0.69,3.40)$ & $1.78(1.19,2.60)$ & $1.85(1.22,2.73)$ & $1.57(1.21,2.02)$ \\
\hline \multicolumn{6}{|l|}{ All-cause death } \\
\hline FDS1: deceased $(n)$ & 37 & 176 & 311 & 168 & 692 \\
\hline FDS1: years & 3441.3 & 5884.2 & 4356.6 & 951.0 & $14,633.1$ \\
\hline FDS1: IR & 1075.2 & 2991.1 & 7138.6 & $17,665.6$ & 4729.0 \\
\hline Non-diabetic: deceased $(n)$ & 84 & 433 & 869 & 698 & 2084 \\
\hline Non-diabetic: years & $14,383.6$ & $25,127.0$ & $20,891.5$ & 5881.9 & $66,284.1$ \\
\hline Non-diabetic: IR & 584.0 & 1723.3 & 4159.6 & $11,866.9$ & 3144.0 \\
\hline $\operatorname{IRR}(95 \% \mathrm{CI})$ & $1.84(1.22,2.74)$ & $1.74(1.45,2.07)$ & $1.72(1.50,1.96)$ & $1.49(1.25,1.76)$ & $1.50(1.38,1.64)$ \\
\hline
\end{tabular}

$\mathrm{AD}$, Alzheimer's disease; $\mathrm{VaD}$, vascular dementia

shown in Fig. 1. The HR for the competing event of death for those with type 2 diabetes vs no diabetes decreased from 1.67 $(95 \%$ CI $1.53,1.82)$ to $1.57(95 \%$ CI $1.44,1.71)$ after adjustment (Table 4).
This pattern of results was mirrored in the models for dementia subtypes. The sdHRs were consistently lower than the csHRs (ESM Table 1). Type 2 diabetes was associated with increased sdHRs for unspecified dementia only (1.34 
Table 2 Age at time of index dementia record, age at death and median survival after the index dementia record

\begin{tabular}{|c|c|c|c|c|c|c|c|c|c|}
\hline Dementia type & Variable & $n$ & $\begin{array}{l}\text { Age at index } \\
\text { dementia record } \\
\text { (years } \pm S D)\end{array}$ & $p$ value & $n(\%)$ & $\begin{array}{l}\text { Age at death } \\
\text { (years } \pm \mathrm{SD})\end{array}$ & $p$ value & $\begin{array}{l}\text { Survival after index } \\
\text { dementia record } \\
\text { (years, 95\% CI) }\end{array}$ & $p$ value \\
\hline \multirow[t]{2}{*}{ All-cause dementia } & Type 2 diabetes & 179 & $81.3 \pm 7.6$ & \multirow[t]{2}{*}{0.004} & $146(81.6)$ & $83.2 \pm 7.3$ & \multirow[t]{2}{*}{$<0.001$} & $0.72(0.11,2.38)$ & \multirow[t]{2}{*}{0.08} \\
\hline & No diabetes & 636 & $83.0 \pm 6.8$ & & $525(82.7)$ & $85.5 \pm 6.4$ & & $1.29(0.16,3.11)$ & \\
\hline \multirow[t]{2}{*}{ Alzheimer's disease } & Type 2 diabetes & 57 & $79.9 \pm 7.2$ & \multirow[t]{2}{*}{0.054} & $48(84.2)$ & $82.8 \pm 6.9$ & \multirow[t]{2}{*}{0.046} & $0.86(0.19,3.42)$ & \multirow[t]{2}{*}{0.017} \\
\hline & No diabetes & 268 & $81.7 \pm 6.3$ & & $204(76.1)$ & $84.8 \pm 6.1$ & & $2.17(0.73,4.24)$ & \\
\hline \multirow[t]{2}{*}{ Vascular dementia } & Type 2 diabetes & 20 & $79.3 \pm 6.5$ & \multirow[t]{2}{*}{0.08} & $16(80.0)$ & $81.8 \pm 5.9$ & \multirow[t]{2}{*}{0.046} & $1.57(0.35,6.22)$ & \multirow[t]{2}{*}{0.82} \\
\hline & No diabetes & 54 & $82.3 \pm 6.6$ & & $49(90.7)$ & $85.4 \pm 6.1$ & & $1.88(0.55,3.34)$ & \\
\hline \multirow[t]{2}{*}{ Unspecified dementia } & Type 2 diabetes & 85 & $82.4 \pm 7.7$ & \multirow[t]{2}{*}{0.13} & $67(78.8)$ & $84.0 \pm 7.6$ & \multirow[t]{2}{*}{0.049} & $0.56(0.13,2.22)$ & \multirow[t]{2}{*}{0.59} \\
\hline & No diabetes & 246 & $83.8 \pm 6.8$ & & $213(86.6)$ & $85.9 \pm 6.7$ & & $0.79(0.18,2.42)$ & \\
\hline
\end{tabular}

[95\% CI 1.05, 1.71] and 1.45 [95\% CI 1.13, 1.85] in unadjusted and adjusted models, respectively) but with significantly increased csHRs for vascular dementia $(1.90$ [95\% CI 1.13, 3.18] and 1.87 [95\% CI 1.11, 3.15] for unadjusted and adjusted models, respectively) and unspecified dementia (1.88 [95\% CI 1.46, 2.40] and 1.92 [95\% CI 1.50, 2.47]) for unadjusted and adjusted models, respectively). There was no increased hazard for Alzheimer's disease attributable to diabetes.

\section{Discussion}

In this matched cohort study, we used administrative health data sets to identify dementia in participants with type 2 diabetes from the FDS1, a representative sample from an urban Australian community [21], and residents without diabetes matched by age, sex and postcode. The main aim was to compare the natural history, incidence and risk of dementia in the two groups. Consistent with previous reviews and meta-analyses [1-4], we found that type 2 diabetes was associated with an increased incidence and risk of all-cause dementia. This was mainly explained by an earlier age at onset of dementia in diabetes, as survival with dementia was not significantly different between the groups. There was also an increased risk of premature death in type 2 diabetes, and the magnitude of the risk estimate for dementia was considerably attenuated when the competing event of death was accounted for.

The adjusted cause-specific relative hazard of dementia in type 2 diabetes (csHR 1.51) was virtually identical to estimates obtained in prior meta-analyses of studies using Cox models [2, 3]. In contrast, the Fine and Gray models, which included the association of type 2 diabetes with the competing event of death, resulted in a smaller relative hazard for dementia (sdHR 1.18). The adjusted HR for the competing event of death (1.57) was of similar magnitude to that for dementia (1.51). In age-related diseases where mortality is high, the competing event of death may obscure the potential risk of dementia had death been avoided. Presentation of the sdHRs of dementia alongside the csHRs of dementia and mortality are necessary to disentangle the effects of mortality on cause-specific dementia rates in individuals with vs without type 2 diabetes $[13,14]$.

To our knowledge, there have been no directly comparable studies of the association between dementia and type 2

Table 3 Models of incident all-cause dementia in the combined cohort of residents with and without diabetes by type 2 diabetes status with age as the time scale unadjusted and adjusted for baseline age, sex, schizophrenia and CCI, using the Fine and Gray model, and Cox model

\begin{tabular}{|c|c|c|c|c|c|}
\hline Dementia type & Baseline variable & $\begin{array}{l}\text { Fine and Gray model } \\
\text { sdHR }(95 \% \mathrm{CI})\end{array}$ & $p$ value & $\begin{array}{l}\text { Cox model csHR } \\
(95 \% \mathrm{CI})\end{array}$ & $p$ value \\
\hline All dementia - unadjusted & Type 2 diabetes & $1.09(0.92,1.29)$ & 0.30 & $1.48(1.26,1.75)$ & $<0.001$ \\
\hline \multirow[t]{8}{*}{ All dementia - adjusted } & Age (/10 year increase) & $1.98(1.79,2.20)$ & $<0.001$ & $0.65(0.57,0.73)$ & $<0.001$ \\
\hline & Male & $0.83(0.72,0.95)$ & 0.008 & $1.06(0.92,1.22)$ & 0.46 \\
\hline & Schizophrenia & $4.07(1.46,11.37)$ & 0.007 & $6.12(2.53,14.79)$ & $<0.001$ \\
\hline & $\mathrm{CCI}$ & & & & $<0.001$ \\
\hline & 0 (reference) & 1 & & 1 & \\
\hline & $1-2$ & $0.79(0.65,0.97)$ & 0.021 & $1.11(0.90,1.35)$ & 0.33 \\
\hline & $3-13$ & $1.05(0.77,1.44)$ & 0.74 & $2.14(1.57,2.91)$ & $<0.001$ \\
\hline & Type 2 diabetes & $1.18(1.00,1.39)$ & 0.055 & $1.51(1.27,1.78)$ & $<0.001$ \\
\hline
\end{tabular}


Table 4 Cox model of the competing event of death in the combined cohort of residents with and without diabetes by type 2 diabetes status with age as the time scale unadjusted and adjusted for baseline age, sex, schizophrenia and CCI

\begin{tabular}{llll}
\hline Model & Baseline variable & Cox model csHR $(95 \% \mathrm{CI})$ & $p$ value \\
\hline Unadjusted & Type 2 diabetes & $1.67(1.53,1.82)$ & $<0.001$ \\
Adjusted & Age (/10 year increase) & $0.58(0.54,0.62)$ & $<0.001$ \\
& Male & $1.59(1.47,1.71)$ & $<0.001$ \\
& Schizophrenia & $2.61(1.40,4.86)$ & 0.003 \\
CCI & & $<0.001$ \\
& 0 (reference) & 1 & $<0.001$ \\
$1-2$ & $1.78(1.62,1.96)$ & $<0.001$ \\
& $3-13$ & $3.08(2.66,3.56)$ & $<0.001$ \\
\hline
\end{tabular}

diabetes that consider the competing event of death, but our findings are consistent with several other related studies. A recent study in older Mexican-American individuals with comparable follow-up to the present study reported lower risk estimates of the association between diabetes and a composite cognitive endpoint (cognitive impairment no dementia plus dementia) with Fine and Gray compared with Cox modelling [16]. The present findings on age at dementia onset, death and survival are also similar to those of a population-based study of incident dementia that employed the same dementia case ascertainment method using WADLS. In this latter study, the presence of any diabetes was associated with a dementia onset and death an average 2.2 and 2.6 years earlier and with significantly but modestly reduced survival times with dementia [18]. The survival times seen in the present and previous study [18] are short in comparison with those given in the literature [35]. This is likely to be explained by a number of factors including the known short survival times seen in late-onset dementia [35], by our use of a proxy age for dementia onset and by our reliance on clinically diagnosed rather than screened cases [36].

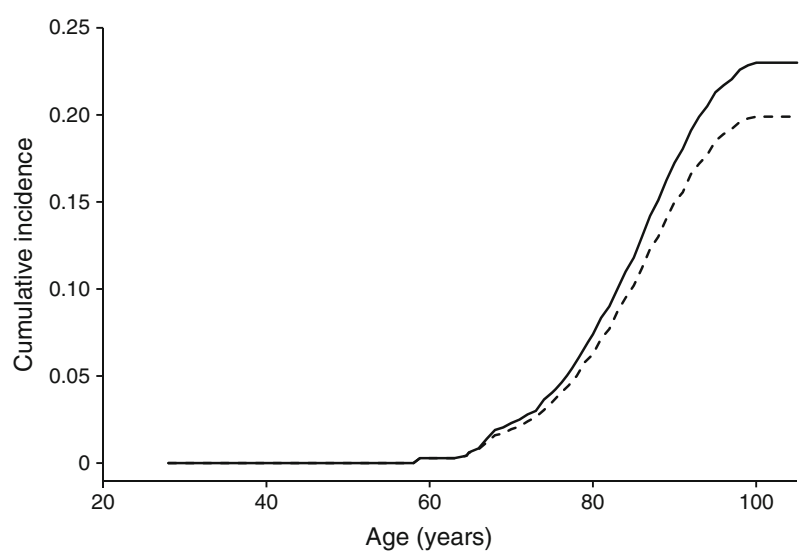

Fig. 1 Cumulative incidence function of all-cause dementia by type 2 diabetes status adjusted for baseline age, sex, schizophrenia and CCI (from the Fine and Gray adjusted model in Table 3; sdHR 1.18, 95\% CI 1.00, 1.39, $p=0.055)$. Solid line, type 2 diabetes cohort; dashed line, age-, sex-, and postcode-matched cohort without diabetes
The present study has clinical and public health implications. First, fear of dementia is common in the general population [37], and this may be greater in people with diabetes who are aware that that they are at increased risk of cognitive decline. Information relating to the probability and time course of dementia could inform communications with patients with type 2 diabetes and help to reduce anxiety [38]. Second, there has been considerable interest in type 2 diabetes as a potential target for prevention of dementia [7], and risk scores for late-life dementia to be used in middle age have been tested [6]. Our finding that diabetes may be a less potent risk factor for late-life dementia may explain why diabetes did not improve the prediction of dementia in one recent model [6]. Nevertheless, a 2 year age difference in dementia onset has public health importance as even a 1 year delay in dementia onset has been estimated to have a major impact on projections for dementia populations [39]. Although there are currently no models to link cognitive trajectories with incidence of dementia, it has recently been demonstrated that type 2 diabetes confers the equivalent of a 2-3 year acceleration of cognitive ageing [40], suggesting that shared pathophysiological processes contribute to cognitive ageing and risk of dementia in diabetes.

We explored dementia subtypes and included these data for interest, but they should be interpreted with caution. Although all cases required a diagnosis of dementia to be recorded by a medical practitioner, the diagnostic criteria and accuracy cannot be determined given the broad-based source of the diagnostic information. Unspecified dementia (40.6\%) and Alzheimer's disease (39.9\%) were the two most frequent subtypes, and vascular dementia was least common (9.1\%). It is possible that the presence of diabetes could reduce the likelihood of an Alzheimer's disease diagnosis being recorded. The dementia subtype data may aid interpretation in that the csHR and sdHR for Alzheimer's disease suggest no significant association between type 2 diabetes and Alzheimer's disease; the sdHR may be slightly lower than the csHR because those with type 2 diabetes are more likely to die before experiencing Alzheimer's disease. For vascular dementia, both the csHR and sdHR were elevated, indicating 
an increased risk in diabetes. Unspecified dementia had estimates similar to those of vascular dementia, suggesting that much of this subtype could be related to vascular dementia or possibly mixed pathology.

The present study has several strengths. It was conducted in a community-based cohort with accurately diagnosed type 2 diabetes that was representative of the urban Australian diabetes community [21] and in carefully matched residents without diabetes, including matching to the level of Australian postcodes. Matching and dementia case-finding used WADLS, an established, well-developed and validated data linkage system. Although the comparison sample could have included individuals with diabetes, none had diabetes coded at any time before or during the study period.

The main limitation of the present study was the use of health administrative data to detect individuals with dementia. However, we used the same method for both participant groups and there is evidence supporting the validity of the approach. In Australia, the sensitivity, positive predictive value and $\mathrm{K}$ value for ICD-10 dementia diagnoses in hospital records were $67 \%, 76 \%$, and 0.71 , respectively, suggesting substantial agreement between medical charts and registry data [41]. We have previously demonstrated that the use of combined databases in WADLS substantially increases the detection of individuals with dementia [26, 28]. In a Danish study, $86 \%$ of dementia and $81 \%$ of Alzheimer's disease diagnoses in hospital registers were correct, while the accuracy for vascular dementia was lower [42].

The other major limitation relates to the potential for survival and selection bias related to the recruitment of the FDS1 cohort who were then matched with residents without diabetes. Such potential sources of bias are likely to result in an underestimate of the association between diabetes and dementia. We had limited ability to adjust for confounders, especially educational status, but matching on postcode may be a surrogate for socioeconomic, migrant and educational status. We were also unable to adjust for other potentially important variables such as diet, exercise, obesity, glycaemia and cardiovascular risk factors, but were able to adjust for comorbidities associated with these risk factors, incorporated into the CCI.

In conclusion, type 2 diabetes is associated with an increased incidence and relative hazard for dementia. Dementia in type 2 diabetes occurs with a younger age of onset compared with community-based residents without diabetes, but has a similar duration of survival. As a consequence of the higher risk of premature mortality, the increased risk of dementia in type 2 diabetes is not as marked as suggested by cause-specific HRs.

Acknowledgements The authors wish to thank the Fremantle Diabetes Study staff, investigators and participants, and the staff at the Western Australian Data Linkage Branch and the MHIS, the HMDS and Death Registrations.
Funding The Raine Foundation, University of Western Australia, provided seed funding for the Fremantle Diabetes Study. The cost of data linkage was supported by the Fremantle Hospital Medical Research Foundation. TMED was supported by a National Health and Medical Research Council of Australia Clinical Practitioner Fellowship.

Duality of interest statement The authors declare that there is no duality of interest associated with this manuscript.

Contribution statement WAD conceived the study design, analysed and researched the data and assisted with the manuscript. RRZ assisted with data analysis, contributed to the discussion and reviewed/edited the manuscript. SES contributed to the study design, assisted with interpretation of the data and reviewed and edited the manuscript. TMED designed the FDS1, contributed to the study design, assisted with data interpretation and reviewed/edited the manuscript. DGB conceived the study design, assisted with data analysis and interpretation, wrote the manuscript and reviewed/edited the final version of the manuscript. All authors reviewed/edited and approved the final version of the manuscript. DGB is responsible for the integrity of the work as a whole.

\section{References}

1. Exalto LG, Whitmer RA, Kappele LJ, Biessels GJ (2012) An update on type 2 diabetes, vascular dementia and Alzheimer's disease. Exp Gerontol 47:858-864

2. Cheng G, Huang C, Deng H, Wang H (2012) Diabetes as a risk factor for dementia and mild cognitive impairment: a meta-analysis of longitudinal studies. Intern Med J 42:484-491

3. Gudala K, Bansal D, Schifano F, Bhansali A (2013) Diabetes mellitus and risk of dementia: a meta-analysis of prospective observational studies. J Diabetes Investig 4:640-650

4. Biessels GJ, Staekenborg S, Brunner E, Brayne C, Scheltens P (2006) Risk of dementia in diabetes mellitus: a systematic review. Lancet Neurol 5:64-74

5. Zimmet PZ, Magliano DJ, Herman WH, Shaw JE (2014) Diabetes: a 21st century challenge. Lancet Diabetes Endocrinol 2:56-64

6. Exalto LG, Quesenberry CP, Barnes D, Kivipelto M, Biessels GJ, Whitmer RA (2013) Midlife risk score for the prediction of dementia four decades later. Alzheimers Dement: J Alzheimers Assoc 10: 562-570

7. Biessels GJ, Strachan MW, Visseren FL, Kappelle LJ, Whitmer RA (2014) Dementia and cognitive decline in type 2 diabetes and prediabetic stages: towards targeted interventions. Lancet Diabetes Endocrinol 2:246-255

8. Matthews FE, Arthur A, Barnes LE et al (2013) A two-decade comparison of prevalence of dementia in individuals aged 65 years and older from three geographical areas of England: results of the Cognitive Function and Ageing Study I and II. Lancet 382:14051412

9. Qiu C, von Strauss E, Backman L, Winblad B, Fratiglioni L (2013) Twenty-year changes in dementia occurrence suggest decreasing incidence in central Stockholm, Sweden. Neurology 80:1888-1894

10. Wu YT, Fratiglioni L, Matthews FE et al (2015) Dementia in western Europe: epidemiological evidence and implications for policy making. Lancet Neurol 15:116-124

11. Gregg EW, Li Y, Wang J et al (2014) Changes in diabetes-related complications in the United States, 1990-2010. N Engl J Med 370: $1514-1523$

12. Davis TM, Hunt K, McAullay D et al (2012) Continuing disparities in cardiovascular risk factors and complications between aboriginal 
and Anglo-Celt Australians with type 2 diabetes: the Fremantle Diabetes Study. Diabetes Care 35:2005-2011

13. Berry SD, Ngo L, Samelson EJ, Kiel DP (2010) Competing risk of death: an important consideration in studies of older adults. J Am Geriatr Soc 58:783-787

14. Koller MT, Raatz H, Steyerberg EW, Wolbers M (2012) Competing risks and the clinical community: irrelevance or ignorance? Stat Med 31:1089-1097

15. Seshasai SR, Kaptoge S, Thompson A et al (2011) Diabetes mellitus, fasting glucose, and risk of cause-specific death. N Engl J Med 364:829-841

16. Mayeda ER, Haan MN, Kanaya AM, Yaffe K, Neuhaus J (2013) Type 2 diabetes and 10-year risk of dementia and cognitive impairment among older Mexican Americans. Diabetes Care 36:26002606

17. Brookmeyer R, Johnson E, Ziegler-Graham K, Arrighi HM (2007) Forecasting the global burden of Alzheimer's disease. Alzheimers Dement: J Alzheimers Assoc 3:186-191

18. Zilkens RR, Davis WA, Spilsbury K, Semmens JB, Bruce DG (2013) Earlier age of dementia onset and shorter survival times in dementia patients with diabetes. Am J Epidemiol 177:1246-1254

19. Murthy SB, Jawaid A, Qureshi SU et al (2010) Does diabetes mellitus alter the onset and clinical course of vascular dementia? Behav Neurol 23:145-151

20. Helzner EP, Scarmeas N, Cosentino S, Tang MX, Schupf N, Stern Y (2008) Survival in Alzheimer disease: a multiethnic, populationbased study of incident cases. Neurology 71:1489-1495

21. Davis TM, Bruce DG, Davis WA (2012) Cohort profile: The Fremantle Diabetes Study. Int J Epidemiol 42:412-421

22. Bruce DG, Davis WA, Casey GP et al (2008) Predictors of cognitive impairment and dementia in older people with diabetes. Diabetologia 51:241-248

23. Bruce DG, Davis WA, Casey GP et al (2008) Predictors of cognitive decline in older individuals with diabetes. Diabetes Care 31: 2103-2107

24. Bruce DG, Davis WA, Starkstein SE, Davis TM (2014) Mid-life predictors of cognitive impairment and dementia in type 2 diabetes mellitus: the Fremantle Diabetes Study. J Alzheimers Dis 42:S63S70

25. Holman CD, Bass AJ, Rouse IL, Hobbs MS (1999) Populationbased linkage of health records in Western Australia: development of a health services research linked database. Aust N Z J Public Health 23:453-459

26. Zilkens RR, Spilsbury K, Bruce DG, Semmens JB (2009) Linkage of hospital and death records increased identification of dementia cases and death rate estimates. Neuroepidemiology 32:61-69

27. Zilkens RR, Spilsbury K, Bruce DG, Semmens JB (2009) Clinical epidemiology and in-patient hospital use in the last year of life
(1990-2005) of 29,884 Western Australians with dementia. J Alzheimers Dis 17:399-407

28. Zilkens RR, Bruce DG, Duke J, Spilsbury K, Semmens JB (2014) Severe psychiatric disorders in mid-life and risk of dementia in latelife (age 65-84 years): a population based case-control study. Curr Alzheimer Res 11:681-693

29. Lawrence D, Hancock KJ, Kisely S (2013) The gap in life expectancy from preventable physical illness in psychiatric patients in Western Australia: retrospective analysis of population based registers. BMJ 346:f2539

30. Charlson ME, Pompei P, Ales KL, MacKenzie CR (1987) A new method of classifying prognostic comorbidity in longitudinal studies: development and validation. J Chronic Dis 40:373-383

31. Lambert TJ, Chapman LH, Consensus Working G (2004) Diabetes, psychotic disorders and antipsychotic therapy: a consensus statement. Med J Aust 181:544-548

32. Lau B, Cole SR, Gange SJ (2009) Competing risk regression models for epidemiologic data. Am J Epidemiol 170:244-256

33. Allignol A, Schumacher M, Wanner C, Drechsler C, Beyersmann J (2011) Understanding competing risks: a simulation point of view. BMC Med Res Methodol 11:86

34. Thiebaut AC, Benichou J (2004) Choice of time-scale in Cox's model analysis of epidemiologic cohort data: a simulation study. Stat Med 23:3803-3820

35. Brodaty H, Seeher K, Gibson L (2012) Dementia time to death: a systematic literature review on survival time and years of life lost in people with dementia. Int Psychogeriatr 24:1034-1045

36. Rait G, Walters K, Bottomley C, Petersen I, Iliffe S, Nazareth I (2010) Survival of people with clinical diagnosis of dementia in primary care: cohort study. BMJ 341:c3584

37. Cantegreil-Kallen I, Pin S (2012) Fear of Alzheimer's disease in the French population: impact of age and proximity to the disease. Int Psychogeriatr 24:108-116

38. Bogardus ST Jr, Holmboe E, Jekel JF (1999) Perils, pitfalls, and possibilities in talking about medical risk. JAMA 281:1037-1041

39. Brookmeyer R, Corrada MM, Curriero FC, Kawas C (2002) Survival following a diagnosis of Alzheimer disease. Arch Neurol 59:1764-1767

40. Tuligenga RH, Dugravot A, Tabak AG et al (2014) Midlife type 2 diabetes and poor glycaemic control as risk factors for cognitive decline in early old age: a post-hoc analysis of the Whitehall II cohort study. Lancet Diabetes Endocrinol 2:228-235

41. Henderson T, Shepheard J, Sundararajan V (2006) Quality of diagnosis and procedure coding in ICD-10 administrative data. Med Care 44:1011-1019

42. Phung TK, Andersen BB, Hogh P, Kessing LV, Mortensen PB, Waldemar G (2007) Validity of dementia diagnoses in the Danish hospital registers. Dement Geriatr Cogn Disord 24:220-228 